\title{
Azoospermia, CTCAE
}

National Cancer Institute

\section{Source}

National Cancer Institute. Azoospermia, CT CAE. NCI Thesaurus. Code C143312.

A disorder characterized by laboratory test results that indicate complete absence of spermatozoa in the semen. 